\title{
Article \\ Surface and Interface Designs for Friction Control
}

\author{
Stephen $\mathrm{Hsu}^{1)^{*}}$, Govindaiah Patakamuri ${ }^{1)}$ and Lawrence $\mathrm{Li}^{2)}$ \\ ${ }^{1)}$ Department of Mechanical \& Aerospace Engineering, George Washington University, USA \\ ${ }^{2)}$ Department of Mechanical \& Biomedical Engineering, City University of Hong Kong, China \\ *Corresponding author: Stephen Hsu (stevehsu@gwu.edu)
}

Manuscript received 15 Februaly 2018; accepted 31 May 2018; published 15 July 2018

Presented at the 6th World Tribology Congress 2017 Beijing, September 2017

\begin{abstract}
Modern engineering surfaces are cut, grind, and polished to create surfaces that are isotropic, uniform, and homogeneous to enable easy assembly and manufacture. Commonly used surface descriptors such as Ra are based on these characteristics. In the 1990s, the magnetic hard disk drive storage industry began to use multiscale directionally aligned roughness to achieve its lubrication requirements successfully, and this leads to the concept of designed surface topography and textures to enable additional functionalities. The design and fabrication of surface topography to impart directionality, uniform reactivity, and patterned geometric dimples or protrusions can enhance or expand the surface properties to meet specific desirable application needs. Adding thin films, coatings, multilayer nanocomposites on the surface can alter the surface physical and chemical characteristics and enhance durability. Properly designed surface textures (dimples, grooves, and discrete shapes) can reduce friction, create drag reduction, as well as control the real area of contact and heat transfer characteristics. In terms of designing friction reducing surfaces for an application, the dominant friction mechanisms need to be understood and proper surface designs applied to reduce the dominant friction generation. Generally, roughness perpendicular to the sliding direction will increase frictional resistance while increasing the interfacial temperatures. Multiscale roughness parallel to the sliding direction, depending on the speed and load of the contact, will reduce scuffing and reduce friction. In this paper, we use surface textures coupled with diamond-like-carbon thin films and bonded chemical layer to effect friction reduction of piston ring-cylinder liner friction.
\end{abstract}

\section{Keywords}

friction control, topography, multiscale textures, surface reactivity

\section{Introduction}

Modern engineering surfaces are designed to be smooth, uniform, and isotropic. These characteristics allow machined parts to be assembled into systems quickly. Surface metrology tools and practice have emerged and evolved to describe the engineering surface since 1945.

During the 1960s, spurred by the space exploration, high precision bearings and rotating interfaces were needed. The smooth isotropic surfaces became the norm for tribological systems. Two industrial problems emerged to challenge this norm: 1) surface defects during metal working processes; 2) diesel engine scuffing between ring and liner. In the metal working process, cutting edge often experienced lubricant starvation, resulting in scuffing and damage. For both issues, micro-dimple surface textures provided solutions. Discrete dimples on the tool surface provided coolant in-situ, and crosshatching grooves with oil reservoir minimized scuffing.

This early innovation in creating "tailored surface" for specific problem solving was, in fact, a preview of the future. The constraint for tailored surface was the cost of fabrication and the lack of known design guideline. So the "surface texturing" incidence was viewed as an expensive special case to solve specific problem. The technical community moved forward to make smooth, uniform surfaces by developing technology for finishing, polishing, and single diamond turning, etc. During the 1990s, the magnetic hard disk information storage industry, in their pursuit of ever higher data storage and speed to support high speed computing, began to experiment with multiscale surface features and multi-layered structures, resulting in new manufacturing processes that were able to control features from nanometer scale to microscales. The availability of this technology expanded the horizon of what constituted "surface engineering". Smooth, isotropic surface was no longer adequate for data storage. In an effort to create self-reassembled lubricant molecular thin film, the surface was textured to create directionally aligned grooves as shown in Fig. 1. The height of the surface ridge was tightly controlled to an average of $5 \mathrm{~nm}$; 


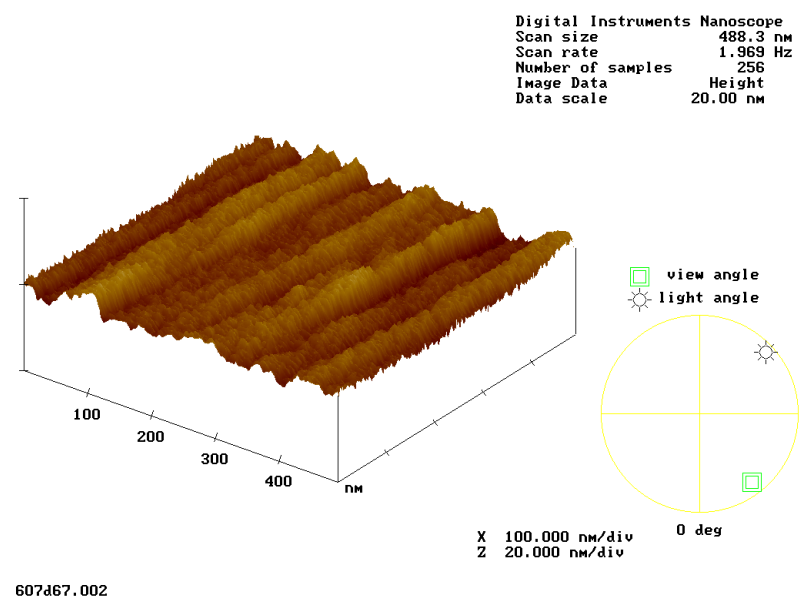

Fig. 1 Directionally aligned surface roughness

enable the use of monolayer of ZDOL lubricant on the hard disk to satisfy the designed 7 years disk life. Using high resolution AFM imaging technique, we examined the lubrication mechanisms of ZDOL. Figure 2 shows the surface topography of a disk with ZDOL. Using AFM-based nanomechanical technique, we measured the nano-scale elasticity of the surface and found most of the ZDOL actually resided in the valley [1] When the slider touched the surface, the molecules migrated to the contact driven by the surface tension force, effecting lubrication. If the amount of ZDOL molecules were sufficient to form meniscus at the contact interface, the slider will tend to stick to the surface from the capillary forces.

The successful demonstration of how surfaces can be engineered from nanometer scale to millimeter scale to achieve specific performance goals has fundamentally changed the perspective of what surface engineering is. The equipment, tools, characterization methods used in the magnetic storage industry have provided the means to seriously consider tailored surfaces with multiscale features, if necessary, to control interfacial properties. However, designing a surface (interface) for friction control is more complex than just designing one surface to achieve a specific property. Sliding interface involves two separate surfaces coming together and the contact mechanics and the sliding friction mechanisms will have to be taken into account. We will first examine the nature of friction.

\section{Nature of friction and the effect of surface roughness}

Friction is a complex phenomenon. It is a real time manifestation of energy dissipation process when loaded interfaces slide over each other. Therefore it is transient by nature. The instantaneous friction force is the results of all the contact events at the interface as well as the events associated with the tribosystem and the environmental. Any perturbations, such as temperature change, humidity, vibrations, contaminations, and alignment will change the nature of the friction force and its magnitude. Under very low load, the contact may have multiscale contact sequences, and the dominant frictional events may shift from nanometer scale events to microscale events and from microscale to millimeter scale [2].

Since the nature of friction is complex and depends on many parameters, the friction mechanisms also depends on many transient factors, designing surfaces to control friction is a huge challenge. At the same time, if we ignore all the intrinsic transient factors and focus on the dominant steady state friction level, the complexity reduces significantly.

To achieve the engineering goal of friction control via surface design, we need to focus on the key steady state friction mechanism and its major contributors the resistance forces. We will define friction in this paper, simply as the total resistance force to motion, which can be easily measured. For multiscale contact case, the dominant length scale that controls the system friction will be assumed. This eliminates heat and photon emission of the energy dissipation process. At nanoscale, surface forces, capillary forces, surface tension, and surface energy all significantly contribute to friction. At microscale, deformation of the asperity-surface interaction is important. At macroscale, plowing is the dominant contributor to friction. Since the basic definition of friction stems from the total resistance force, the dominant force at whatever scales will dominate the resulting resistance force. This will clarify the confusion on the scaling effect on friction. Therefore, in designing surface and interfaces for friction control, the magnitude of the various forces at different scales will be compared to facilitate which scale is important to control, at least for the first approximation.

It is generally recognized that surface roughness influences friction [3-5]. Other parameters such as contact geometry, relative hardness, elastic modulus, lubrication, load, speed, and the environment all play a role in shaping the final resistance (friction). The general observation is rough surface increases friction.

Friction is generated when one surface slides against the other. Generally the sliding can be classified into two regimes: 1) no asperity penetration zero wear friction regime; 2) asperity penetration and the load is supported by asperity contacts. For the first case, if the surfaces are flat, isotropic, and non-adhesive, then the resulting friction is primarily come from the surface forces interactions. To check our hypothesis, we conducted

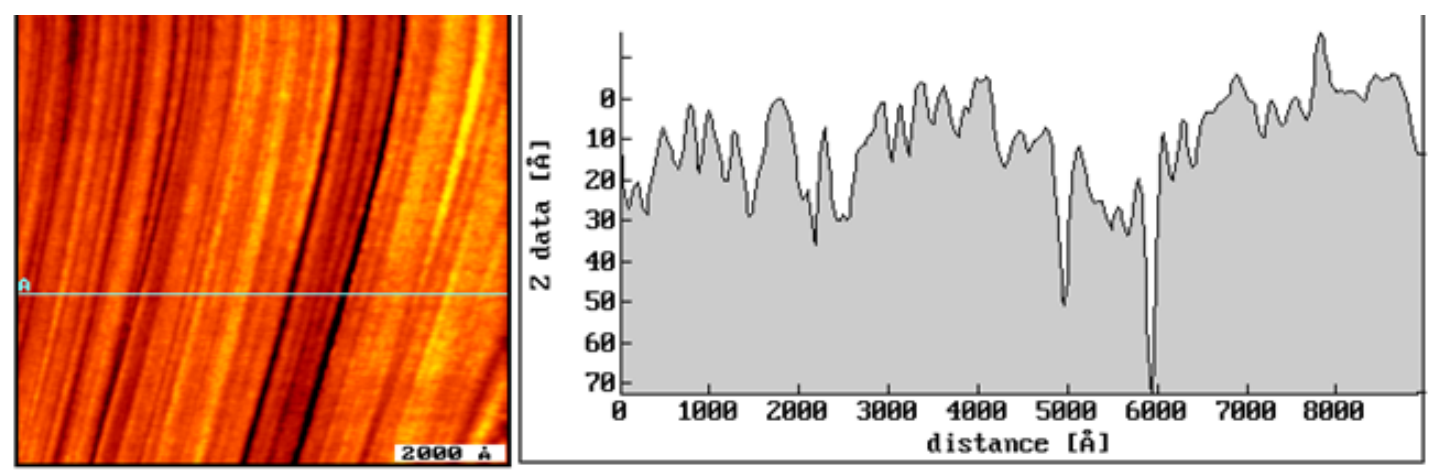

Fig. 2 Topography of a hard disk with a manolayer of ZDOL [1] 
simple experiments using a Hysitron Triboindenter to measure friction at nano-, micro-scales. The Triboindenter has rigid and stiff frame with capacitance force sensors for accurate force measurements. The $\mathrm{x}, \mathrm{y}, \mathrm{z}$ force sensors also ensure that the simple sliding is conducted at fixed height and maintain a constant direction. This is important to obtain accurate friction. One of the issues in this experimental design is the size, shape, and 3-D characterization of the tip to understand the plowing mechanisms. A diamond tip was used to slide over silicon wafer or fused silica at various loads to examine the friction behavior and compare friction in the two regimes identified above.

Simple sliding friction measurements were conducted using the Triboindenter. The materials used in this study are diamond tips and fused silica (CVI Laser PW-0508-UV) and Si (100) (n type, phosphorus doped, $10^{2} \mathrm{ohm}-\mathrm{cm}$ ). The diamond tips are conical in shape with nominal spherical tip diameters of $0.5 \mu \mathrm{m}$, $1.2 \mu \mathrm{m}$, and $4 \mu \mathrm{m}$. The substrate and tips were cleaned using pure ethanol, dried with nitrogen before use. Experiments were conducted under dry nitrogen atmosphere to minimize humidity influence on silicon-based surface. Potential wear of the diamond tips was checked with friction tests at low loads before and after the high load tests, and the results were repeatable within $\pm 5 \%$, suggesting negligible wear.

The shape, size, and orientation of the tips are crucial parameters for quantitative study of plowing. Accurate measurements of these geometric parameters were achieved using a digital "replica" method.

The diamond tip was pressed onto a $\mathrm{CaF}_{2}$ crystal surface at several locations under various loads to achieve several penetration depths. The impressions were subsequently imaged using an atomic force microscope (AFM). A replica of the tip was obtained by digital inversion of the AFM image of the impression. The replica method provides detailed data on tip symmetry or asymmetry, surface topography and tip orientation if the tip is not symmetrical.

Figure 3(a) shows a replica of a tip that has nearly spherical shape with radius $R=1.2 \mu \mathrm{m}$. The tip surface is smooth, actually with roughness no more than $3 \mathrm{~nm}$ or $0.25 \%$ of the tip radius. Based on the data, the tip is relative symmetrical. The digital reconstructed image of another spherical tip (nominal radius of $4 \mu \mathrm{m}$ ) is shown in Fig. 3(b). This tip is not symmetrical and it resembles an ellipsoid. The long axis in the surface plane, having a radius of $6.4 \mu \mathrm{m}$, is oriented at an angle of $45^{\circ}$ from the $y$ axis, the direction of the tip sliding. The radius of the short axis in the surface plane is $2.8 \mu \mathrm{m}$. Three tips were used in the experiment and they are all characterized.

A series of experiments was conducted to measure the interfacial friction of diamond on silicon and silica under controlled environment. Three diamond tips with nominal diameters of $0.5 \mu \mathrm{m}, 1.2 \mu \mathrm{m}$, and $4.0 \mu \mathrm{m}$ were used but in the data analysis, they are corrected by the tip replica characterization results.

The friction forces as a function of load between the diamond tips and fused silica and silicon substrate were plotted in Fig. 4. The data for the two spherical tips with radii $R=0.5$ $\mu \mathrm{m}$ and $1.2 \mu \mathrm{m}$ can be clearly divided into two regions. In the first region (low-load region), the friction force shows a linear dependence on the load. While in the second region (high-load region), the friction force deviates from the linear relationship and becomes higher with increasing load. Nanoindentation and topographic studies of the substrate surfaces after friction measurements reveal the nature of this friction transition: from
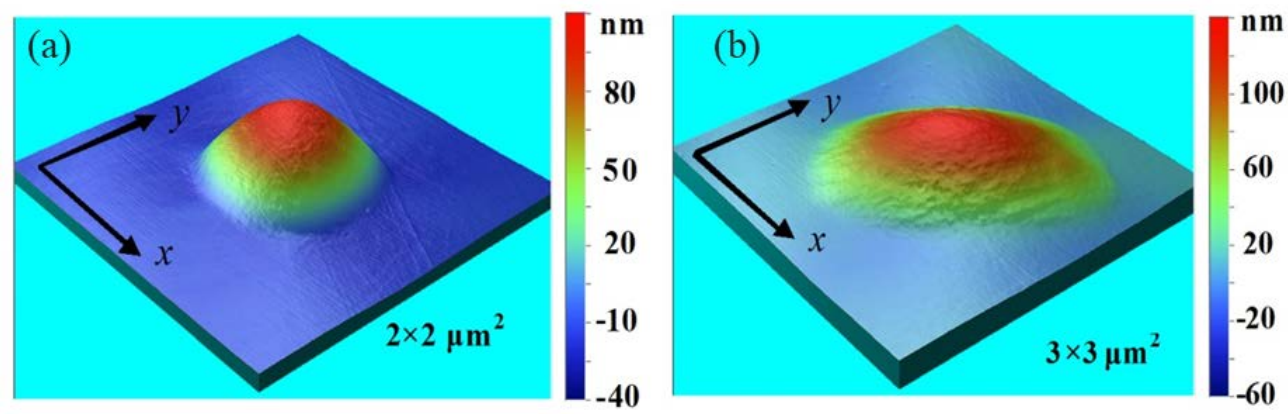

Fig. 3 Geometry of diamond tips used during friction measurements. (a) A spherical tip with radius $R=1.2 \mu \mathrm{m}$,

(b) A tip with a nominal value of radius of $4 \mu \mathrm{m}$ but actually having an ellipsoidal shape
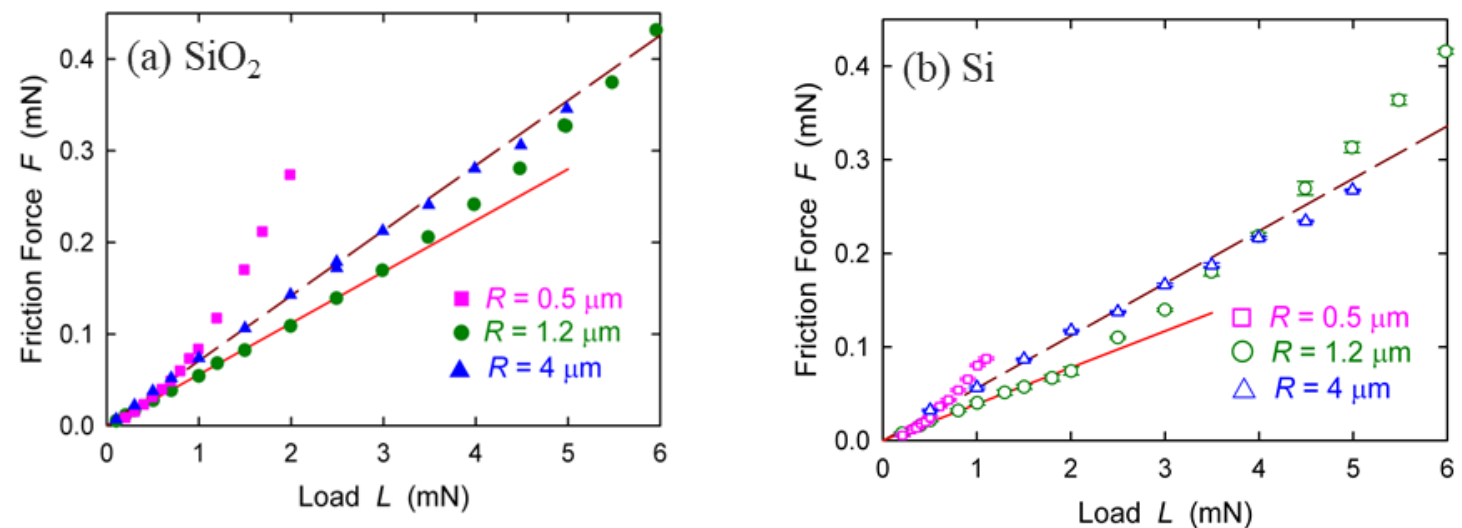

Fig. 4 Friction force as a function of load using three diamond tips with different radii R. Fig. 4(a) uses fused silica substrate and Fig. 4(b) uses silicon substrate 

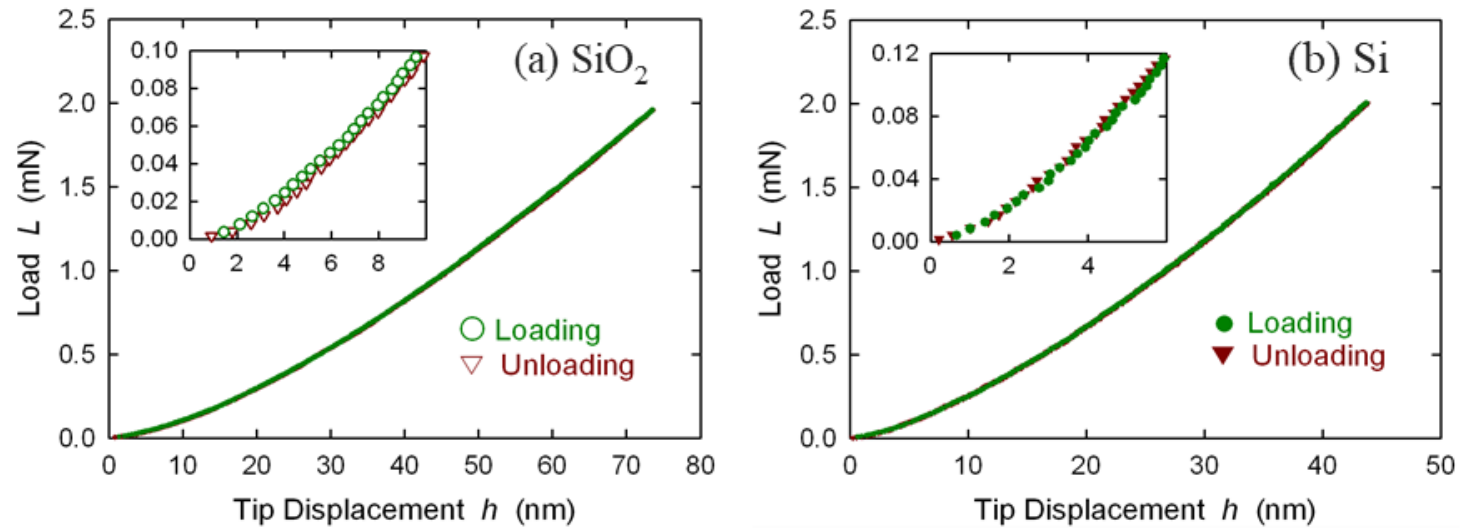

Fig. 5 Load versus displacement data measured during a single indent on silica and silicon using the tip with radius $R=1.2 \mu \mathrm{m}$

elastic deformation to plastic plowing with increasing load.

As seen in Fig. 5, when a low load of $2.0 \mathrm{mN}$ was applied, the loading and unloading curves of for both silicon and fused silica substrates are well overlapped and free of any pop out. Moreover, the wear grooves are too shallow $(\sim 0.4 \mathrm{~nm}$, near the noise level of the measurement) to be discerned, in contrast to nanometers deep grooves at higher loads where plastic deformation occurs, as shown in Fig. 6.

The elastic region shows a linear relationship between friction force $F$ and load $L$

$$
F=\mu_{0} L
$$

Where $\mu_{0}$ is COF and the subscript denotes the elastic region. A common value of $\mu_{0}=0.055 \pm 0.002$ is observed for the two spherical tips on silica substrates, Fig. 4 (a). The plastic region exhibits friction force higher than $\mu_{0} L$, load-dependent COF

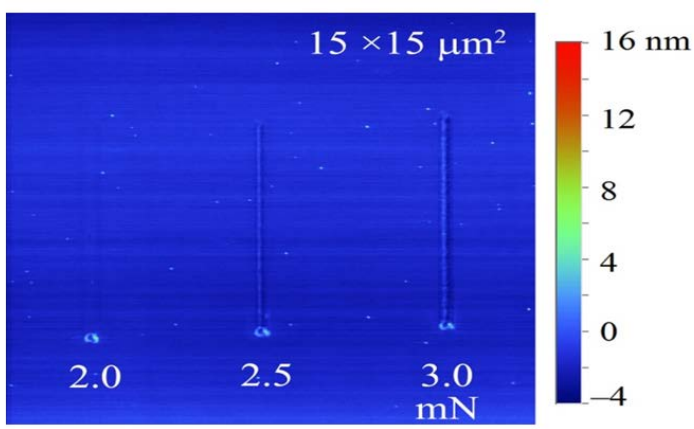

Fig. 6 AFM image of scratch marks on Si (100) at various loads using tip with radius $R=1.2 \mathrm{um}$

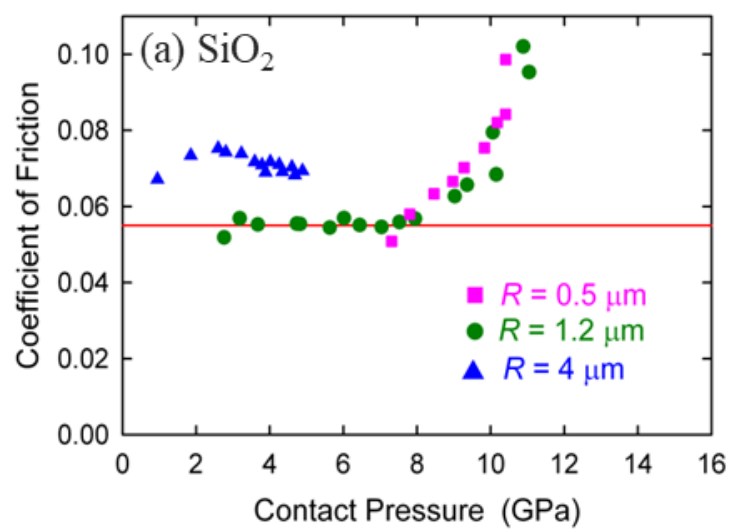

value, and permanent groove created on the substrate surface. The onset load values of the plastic region are $0.5 \mathrm{mN}$ and 4 $\mathrm{mN}$ for $R=0.5 \mu \mathrm{m}$ and $1.2 \mu \mathrm{m}$, respectively. The friction data obtained using the ellipsoidal tip with a nominal radius value of $4 \mu \mathrm{m}$ are all in the elastic region. But the COF value is higher, $0.072 \pm 0.003$.

The friction data for the silicon substrate, Fig. 4(b), are qualitatively similar. The COF value in the elastic region is $0.039 \pm 0.002$ for the two spherical tips. A higher value of $0.056 \pm$ 0.003 is seen for the ellipsoidal tip. The onset load values of the plastic region are $0.4 \mathrm{mN}$ and $2.5 \mathrm{mN}$ for $R=0.5 \mu \mathrm{m}$ and $1.2 \mu \mathrm{m}$, respectively.

The data presented in Fig. 4 clearly shows the different onset load values of the plastic region for different tips. The onset of plastic yield in indentation is described by a yield stress or yield pressure, so the different onset load values for different tips can be explained in terms of contact pressure, which could be calculated from the load and contact area $A_{c}$. Direct measurement of real area of contact is difficult. This is especially true for friction in the elastic region because the material recovery after the friction experiments. In this study, we computed the contact area for the two spherical tips from the experimentally measured tip radius (given by the 3D replica image) and tip penetration depth, and crosschecked it with a direct experimental measurement. The details of the calculation and experimental verification are given in reference [6].

Figure 7 re-plots the COF data shown in Fig. 4, as a function of tip contact pressure. In contrast to different dependences for the two spherical tips shown in Fig. 4, the COF data for these two tip sizes now merge into a single curve in Fig. 7. The

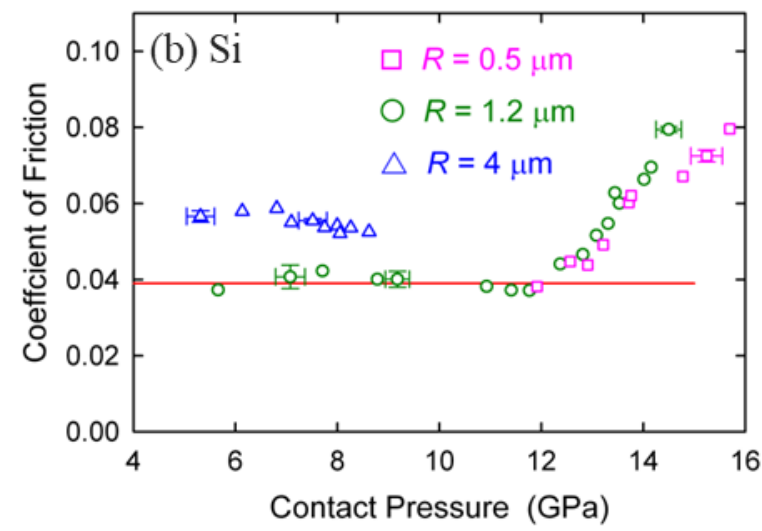

Fig. 7 Coefficient of friction as a function of contact pressure 
plastic region starts at contact pressures of $\sim 9 \mathrm{GPa}$ and $\sim 12$ GPa for fused silica and silicon, respectively. These values are independent of tip size and consistent with the hardness values of these materials.

Figure 7 clearly shows that the high contact pressure at the tip apex induces plastic plowing which introduces additional resistance to the tip motion, i.e., higher COF. Plastic plowing by a moving tip is a phenomenon of plastic flow of materials. It creates a continuous groove on the surface of substrate materials, with pile up of the materials along the sides and at the end of the tip travel path. It is a complex dynamic process in terms of materials response to stress. As a tip penetrates into a substrate and starts to slide horizontally, substrate material is pushed forward and accumulated in front of the tip. As the tip slides further, the material accumulated in front of the tip flows sideway around the tip, forming side pile up [7] (video recording of plastic plowing). The process reaches steady state and excess material forms ridges on the side of the groove.

We have derived an equation to calculate the increased friction force due to plowing.

\section{Global frictional resistance $=$ interfacial friction + plowing friction}

The plowing contribution to friction could be estimated from the material hardness and displaced volume. Details of the verification process are presented in reference [6]. Friction is a phenomenon with relative motion between two contact surfaces in the lateral direction. So contact area in the lateral direction, it is the projected cross-sectional area of the penetrated portion of the tip in the substrate in the sliding direction. It means that the friction force in the elastic region should be proportional to the projected cross-sectional area for a spherical tip. The proportional constant is dependent on the coefficient of friction (COF) in the elastic region and reduced modulus, but independent of tip size. It has a physical meaning as the lateral pressure as the tip slides through the substrate surface elastically.

Plotting of friction force versus projected cross-section area is shown in Fig. 8 for silica and silicon substrates. A linear relationship is observed for both systems and for the two spherical tips with radii $R=0.5 \mu \mathrm{m}$ and $1.2 \mu \mathrm{m}$. With the 3D profiles of the tips obtained using the replica method, we were able to compute projected cross section area for an arbitrary tip shape, which was done for the ellipsoid tip that has a nominal tip radius of $4 \mu \mathrm{m}$. As shown in Fig. 8, the data from this tip follow a common line with that of the spherical tips for each substrate.

The behavior of the friction within the elastic limit could be explained by a model, which recasts the Amontons' law into a form showing frictional force to be proportional to the projected cross-sectional areas in the direction of sliding. This dependence on the cross-sectional area reveals that this is a volumetric dependence instead of just the contact area in the normal direction as commonly assumed by others. The volumetric dependence includes depth of penetration, irregular tip shape, and movement during sliding. All these factors have to be taken into account before the data fall into line. This would suggest that stored elastic strain energy during sliding is a significant part, if not the dominant part of the frictional force in the elastic regime. This observation also experimentally verifies the concept proposed by Ref. [8] that Nano behavior can be achieved from either tip size or depth of penetration perspective. To sum up, the dependence of COF on tip geometry, as well as plastic plowing in the high load condition, are likely to be the major causes of observed high friction values due to plowing.

Experimentally, using a nanoindenter scratching across the surface, including elastic plowing by the tip, the COF generally lies in around 0.03-0.05, since the surface forces contributions to friction are relatively small on non-adhesive surfaces [6]. When the tip enters plastic deformation, the COF immediately increases above 0.05 [6]. If the surfaces are rough, then the asperity plowing will take place, resulting in higher frictional resistance. Alternatively, as often observed in boundary lubrication [9], in the centerline location, where the normal force is the highest, the resulting edge stresses at the entrance of the sliding often cause wear particles to detach from the surface, which can be caused by delamination or fatigue cycles. The wear particle will then abrade the counter-surface creating a groove. This causes loading imbalance from the normal load on the contact. The production of particles will migrate towards the less stressed locations. The resistance force from plowing can be approximated by the volume of material displaced multiplied by its hardness. In most cases, plowing will be the dominant friction contribution. This is the "roughness effect".

\section{Design guideline for friction control}

There are three approaches to design surfaces for friction control: 1) passive friction control to avoid high friction; 2) use surface textures to generate hydrodynamic/hydrostatic pressures to lower the normal force hence reduces friction; 3) the use of coatings, thin films, bonded films to modify the surface properties that will be more amendable to low friction. Surface topography: To avoid plowing friction, smooth homogeneous surfaces such as super-finished surface will be
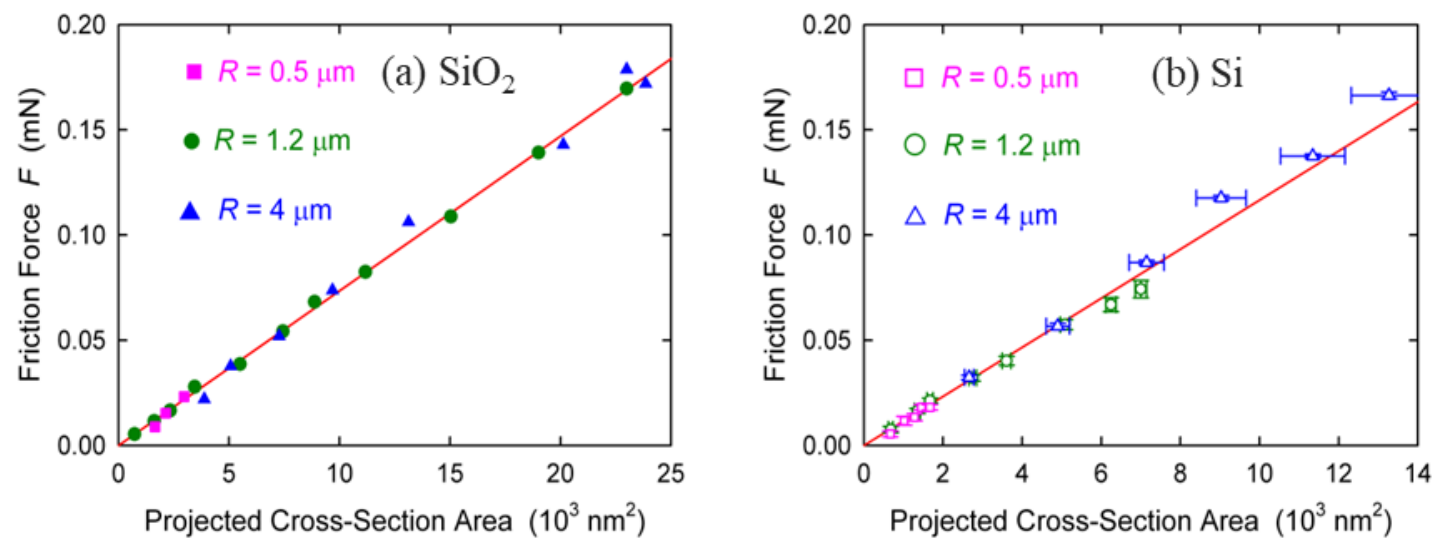

Fig. 8 Friction force as a function of projected cross-section area in the tip sliding direction. Only the data in the elastic region are presented 
desirable. The material pairing is also important, oftentimes, one ignores the deformation friction, even in elastic regime, there is elastic deformation resistance as shown in the simple scratch test results. Plastic deformation also increases resistance to motion. Fatigue wear particles scratching the surface produce plowing friction, then the surface materials should be made to avoid cyclic high stresses which prone to produce delamination, followed by plowing.

Surface textures: such as dimples, protuberances, grooves (such as cross-hatching of engine cylinder liner), multiscale textures, mixed geometric shapes, all can be fabricated on surfaces to achieve specific functionality and alter the surface properties [10-13]. The primary functions are wear debris trapping, generating hydrodynamic pressures, generating hydrostatic pressure, lubricant reservoir, lubricant flow and residence time control for cooling, induce cavitation to generate lift force, etc.

Coatings, thin fims: low friction coatings such as diamond-like carbon (DLC), solid lubricant coatings, self-lubricating coatings, organic-inorganic layer thin films, are all effective under certain conditions for specific applications [13].

All of these tools, inevitably, add cost to the component. So cost-benefit ratios become the determining factor.

\section{Application example of surface texture, DLC, and chemical boned films}

Given the surfaces are smooth, flat and isotropic, if we want to reduce friction, we will need to reduce plowing contribution from either asperities or wear particles. This would suggest that we start with a polished smooth surface at the beginning. Lubricant will be used if feasible which will enable to use of surface texture designs to reduce friction. If lubricant cannot be used, then the use of thin film such as diamond-like-carbon at the appropriate thickness will be good to achieve friction reduction. Two dimensional materials such as graphene or solid lubricant at the thickness at or below the relative surface roughness would also be desirable to achieve low friction.

If liquid lubricant can be used, then the viscosity of the lubricant and the high temperature high shear properties appropriate for the desired operating conditions will need to be specified. The lubricant formulation should contain friction modifiers, depending on the duty cycle and operational severity, molybdenum-containing friction modifiers should be used in conjunction with organic friction modifiers to ensure that friction control can cover all lubrication regimes.

4.1 Design principle for lubricated contacts

Lubrication, to some extent, is the use of the squeezed lubricant film pressure to support the normal load if possible. Under high speed low load conditions, if the fluid pressure generated can support the load, this is called the hydrodynamic lubrication regime. When the load increases or the speed decreases, the fluid pressure may not be sufficient to support the load entirely, allowing the asperities of the surfaces to come into contact, supporting some of the load, this is called the elastohydrodynamic lubrication regime. When the load is very high and the speed is low, the asperity contacts supporting the entire load, boundary lubrication sets in.

The design principle for surface texturing is to generate fluid pressure inside the sliding interface to the maximum extent possible by using: 1) hydrodynamic pressure generation within a dimple; 2 ) by inducing cavitation to create positive pressure; 3 ) by utilizing the deformation of the surface to generate hydrostatic pressure to counteract the compressive load. The hydrodynamic pressure and hydrostatic pressure generation depends on the physical properties of the lubricant, such as bulk modulus, viscosity at the operating temperatures, and the amount of elastic and plastic deformation imposed by the load on the surfaces.

\subsection{Interfacial materials properties requirements}

In order to successfully generate the necessary fluid pressures in a sliding contact, the contact mechanics around the discrete textural features (dimples) is an important factor. In order to seal the dimple, edge stresses generated under loaded sliding around the dimple is needed to seal the lubricant pool. Therefore, rough porous materials or low elasticity material around the dimple will not provide sufficient sealing effect to seal the lubricant pool to generate hydrodynamic or hydrostatic pressure to reduce the normal loading, hence reducing friction. This requires that the materials interface to have a minimum elasticity to allow the edge stress to develop sealing the lubricant inside the dimple [14,15].

\subsection{Areal density of dimples and fluid dynamics}

Since the basic design principle is to generate fluid pressure and hydrodynamic/hydrostatic reaction forces from the transient sliding contact, it is necessary to induce sealing the dimple using the edge stresses. There are misconceptions on textural design: texturing requires conformal contacts; flat contacts are needed; only one surface can be textured, etc. These concepts mostly stem from the successful application of surface textures in the seals area, which operates mostly under hydrodynamic lubrication. The edge stresses (as shown in Fig. 9) are needed for the textural design to function under EHL/BL lubrication regimes.

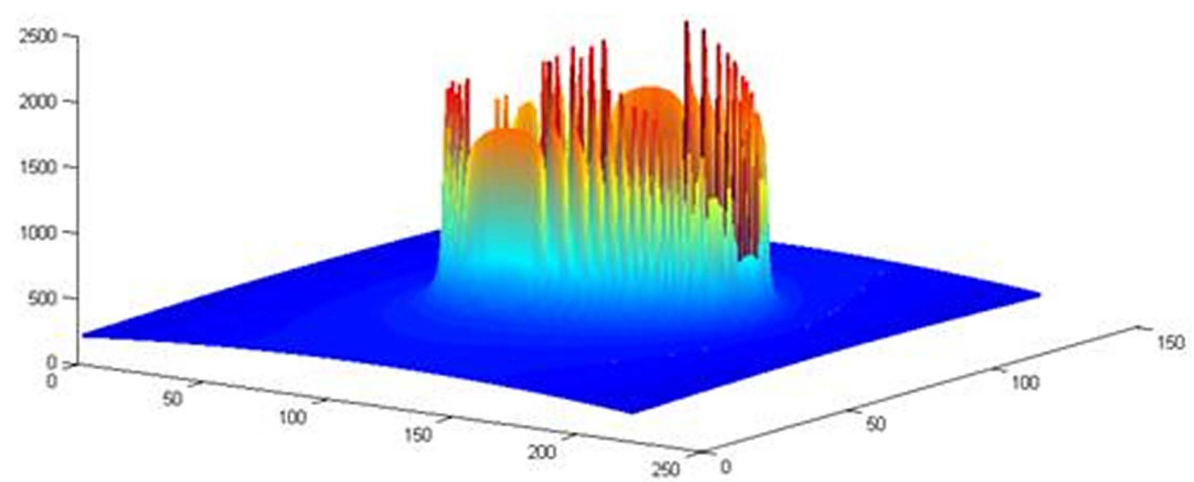

Fig. 9 Edge stresses surrounding an elliptical dimple under boundary lubrication conditions 
While edge stress is essential for effective lift force generation via the texture design, they also introduce a "roughening" effect. The edge stresses alone without generating lift force will increase friction. So this is a balance act via texture pattern design for specific material pair and specific duty cycle and kinematics of motion. Therefore, the areal density (area of textured contact surface over the total contact area) and size of the dimples become an important parameter to achieve friction reduction.

\section{Application of design principle for piston rings}

The ring and liner in this example is from a diesel engine typical of current production. Figure 10 shows the liner with its cross-hatched grooves and the piston ring roughness.

The composite roughness of the two contacting surfaces is about $0.48 \mu \mathrm{m}$. Surface textures were fabricated on the ring surface (crown and its sides) using a mixture of circles and ellipses at an area density of about $10 \%$. The optimum pattern was determined by experimental design. The effectiveness of the texture was measured using a Plint TE77 ring-liner simulator at RT using paraffins with oxidation inhibitors. When the test temperature was raised to $100^{\circ} \mathrm{C}$, the friction jumped, as shown in Fig. 11.

The jump in friction as temperature rises is due to the thinning of the lubricant at high temperatures, which increases
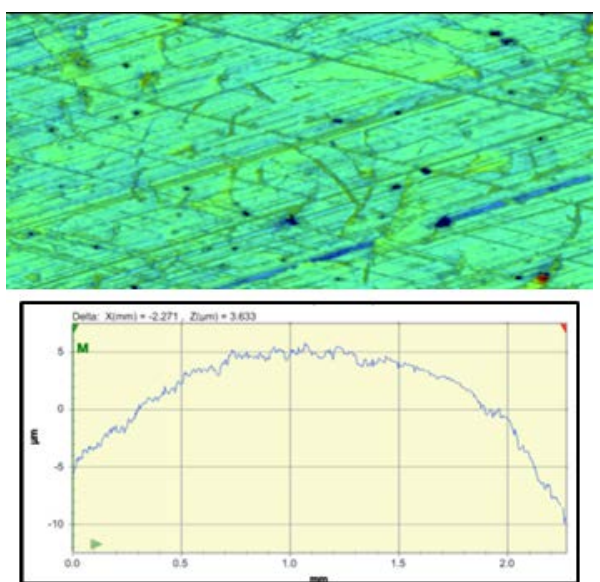

Fig. 10 Cylinder liner with its cross-hatched grooves and the piston ring roughness

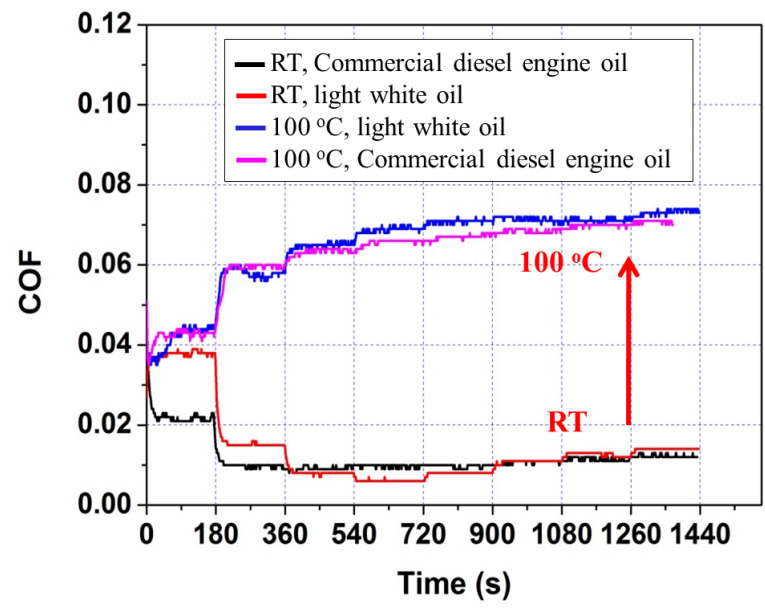

Fig. 11 The effect of temperature on ring liner friction surface contact and friction increases as a result. While the lubricant can be changed to lubricants that have high viscosity under high operating temperatures to maintain low friction, the option may not be available. The issue then becomes how the material technology can be used to effect friction control.

\subsection{The use of diamond-like-carbon thin films}

Diamond-like-carbon (DLC) thin films have been used in engines to reduce friction. DLC is a generic term describing a class of DLC films. Individual DLC films can vary in deposition techniques, doping, compositions, and thickness, resulting in a large range of hardness, elasticity, density, and compositions. Coupled with coating thickness and adhesion characteristics, the variation in performance is very large. Generally, nanohardness and the deposition technique are used to denote a specific composition.

As a result, the friction reduction mechanisms are not clear. DLC properties can vary widely. We chose a medium hardness and were able to deposit a very thin DCL film on the ring surface. Since the DLC is covering the textured surface over a curved ring surface (there ring also has a crown that slides over the grooved liner), the DLC thickness was chosen at $50 \%$ of the relative surface roughness, this way, the surface roughness will become the protector of the thin DLC film from tensile cracking. In applying ultra-thin DLC films below the relatively surface roughness of the engine component surfaces (relative roughness of ring and cross-hatched liner is $0.45 \mu \mathrm{m}$ ), the surface roughness of the steel surface will tend to protect the DLC film, as well as at such thin film state, the film tend to have much more elastic plastic characteristics, prolong film life.

\subsection{Lubrication of DLC}

Since DLC is hard and brittle and it does not react chemically with the antiwear additives in lubricants, we decided to fabricate a bonded chemical film consists of species that are known to react with the dangling bonds of carbon. To help to prevent it from evaporating, we spin coat a polymer monolayer on top, the total thickness is about 2-5 nm (Fig. 12). This organic thin film provides an elasto-plastic layer to resist scratching.

\subsection{Durability evaluation}

Durability of surface textures is an important issue. When surface textures are fully effective, the coefficient of friction can be as low as 0.02 or below. Under such low friction, the contact is fully supported by fluid film pressure, and the likelihood of wear and damage is small. Under start and stop cycles, however, the surfaces may come into contact due to low speeds

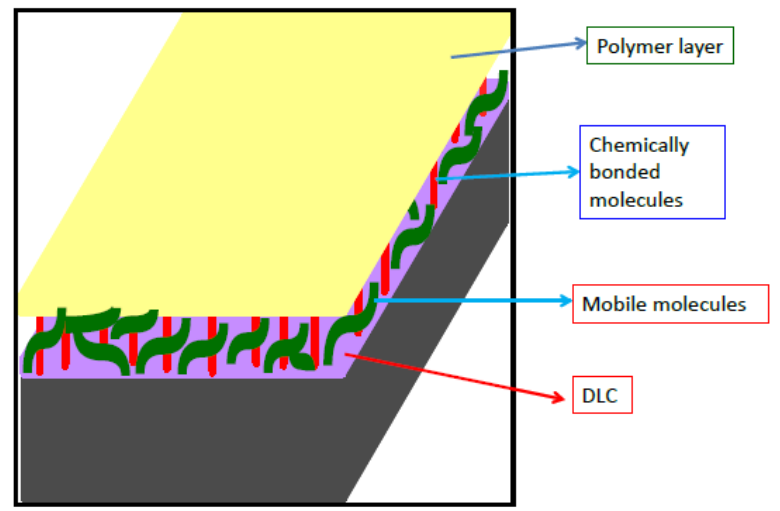

Fig. 12 Bonded chemical film on DLC surface 
and high loads. To alleviate potential wear and damage, thin DLC films, and bonded chemical films which contain DLC reactive chemical species have been used to protect the surface textures as well as to maintain low friction under extreme contact conditions. This kind of testing will require high loads. For this reason, we chose the Four Ball Wear Tester with ball on three flats contact geometry. This is necessary since the four ball contact geometry is difficult to fabricate surface textures and the rapid wear in will certainly wipe out the textures. The ring and liner contact geometry mostly operates under EHL conditions under high load slow speed conditions.

The durability test procedure using a ball-on-three flats contact geometry of a Four Ball Wear Tester is divided into several stages.

Stage I: Minicycle step loading sequence:

First cycle: start at $1.15 \mathrm{~m} / \mathrm{s}$ linear speed (3000 rpm), increase loads from 5, 10, 15, 20, 25, $30 \mathrm{Kg}$ per every 3 minutes (or longer until a steady state friction trace is obtained). The typical time is 3 minutes for each load. Second cycle: lower the speed from $1.15 \mathrm{~m} / \mathrm{s}$ to $0.96 \mathrm{~m} / \mathrm{s}(2500 \mathrm{rpm})$ and repeat the loading from $5 \mathrm{Kg}$ to $30 \mathrm{Kg}$ steps. For a total cycle, the speed changes from
$1.15 \mathrm{~m} / \mathrm{s}, 0.96 \mathrm{~m} / \mathrm{s}, 0.77 \mathrm{~m} / \mathrm{s}, 0.57 \mathrm{~m} / \mathrm{s}, 0.38 \mathrm{~m} / \mathrm{s}$, to $0.19 \mathrm{~m} / \mathrm{s}$ in an increasingly severe test condition (low speed will not allow the textures to generate sufficient hydrostatic lift force, therefore more severe). Minicycle 7 and 8 will basically repeat the condition of cycle 1 and 2 (Fig. 13).

Stage II: Durability test cycle (time to failure test sequence)

To clearly separate the various chemistries and textures, a time to failure test sequence is used. After the 8 minicycles of testing, if no failure is observed, then the test starts at $1.15 \mathrm{~m} / \mathrm{s}$ speed and $2 \mathrm{Kg}$ load for 3 minutes, the load is increased to 5, 10, $20 \mathrm{Kg}$. At that time, the test continues for one hour until failure. If no failure is observed, the load is increased to $30 \mathrm{Kg}$ for an hour, then $40 \mathrm{Kg}$ for an hour (Fig. 14).

\subsection{Durability test results}

Following the above mentioned test procedure, if no failure was observed, the sample was subjected to stage two durability test as illustrated in Fig. 14. As can be seen from Fig. 13, a typical time to failure friction trace shows a sudden friction increase after a long steady friction level. When data from various test sequence are combined, they can be represented in

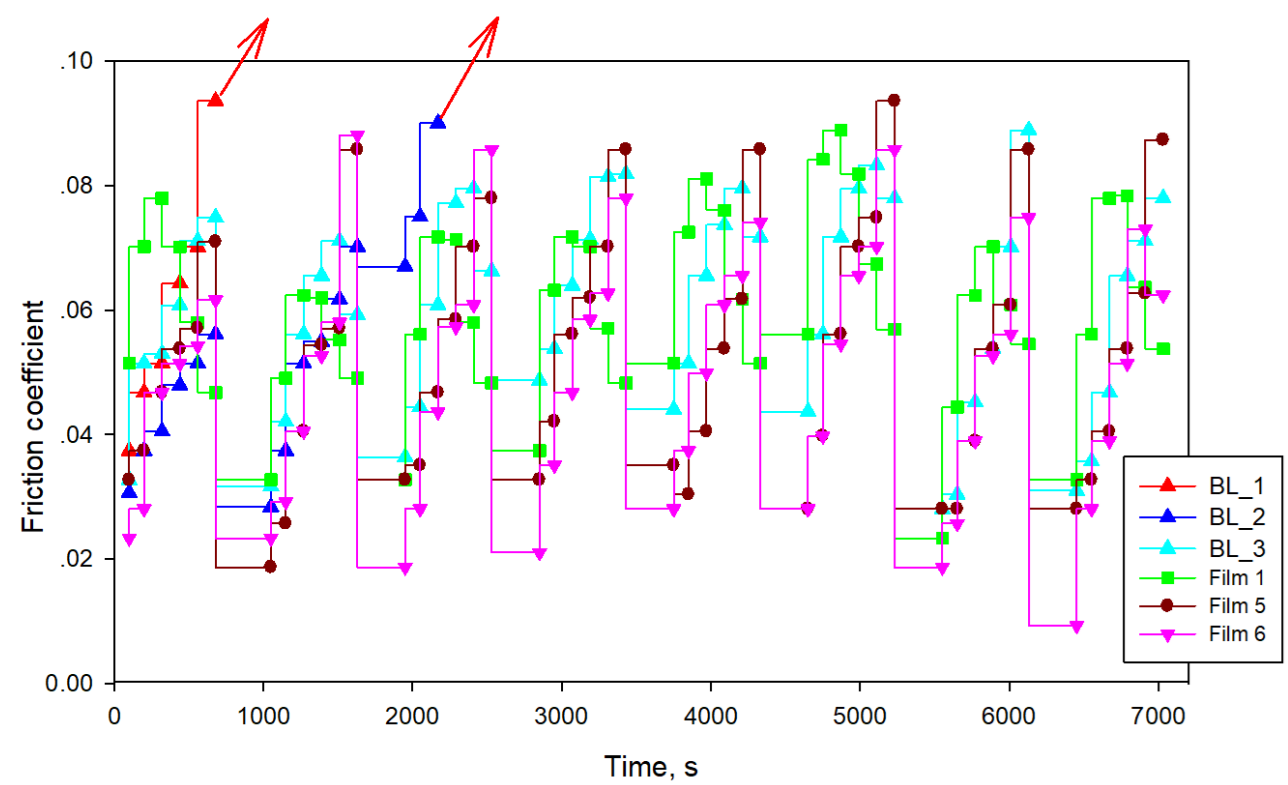

Fig. 13 Test results of various bonded chemical films undergoing the cyclic step loading mini-cycles. Baseline cases failed early as indicated by red arrows. Simple circular dimples were used in $\# 5$ and $\# 6$. No dimples were used in tests $1,2,4$. The dimples are effective in high speed low load conditions

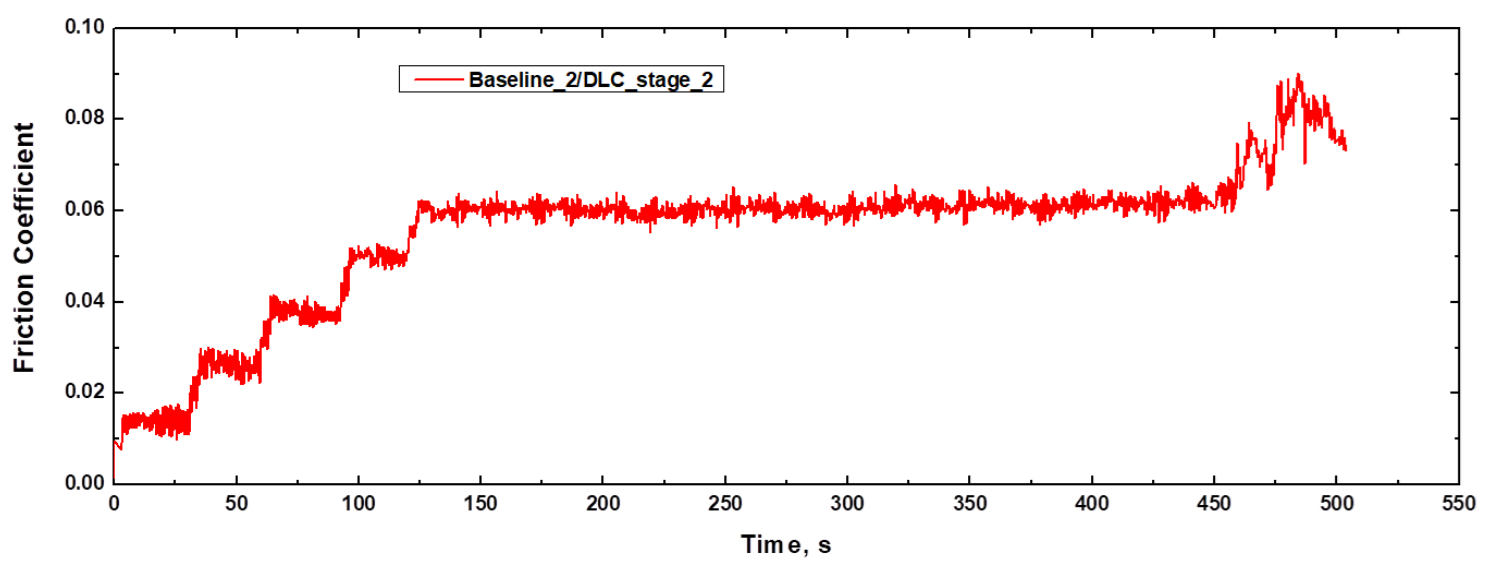

Fig. 14 Time to failure for baseline 2 using a DLC reactive chemical 
two figures (Figs. 15 and 16).

As can be seen from Figs. 15 and 16, after the samples have gone through the 7000 seconds of stage I testing, the test continues at $20 \mathrm{Kg}$ and $3000 \mathrm{rpm}$ speed for an hour. The baseline case using synthetic motor oil without bonded chemical film failed during the $30 \mathrm{Kg}$ load at about 13000 seconds. Film 1 continued to $30 \mathrm{Kg}$ load for one hour, $40 \mathrm{Kg}$ load for another hour, and $50 \mathrm{Kg}$ load for an additional hour without failure. Films 5 and 6 exhibited lower friction and appeared to be able to continue for much longer time. We then stopped the test at the end of $30 \mathrm{Kg}$ load cycle. The concept of built-in bonded film has been successfully demonstrated.

In the same bench cyclic loading sequence test mentioned above, the durability of the textured system extends an order of magnitude over unprotected textured system. Why such small addition of an elasto-plastic layer has such large impact, the answer appears to point to the fact that when the average coefficient of friction under steady state running conditions is between 0.02 to 0.03 , the contact is running under full film lubrication and there is no surface contact, hence no wear. So the only time that wear may take place is during start-stop cycles, and the bounded thin organic layer allow sufficient lubrication and avoid surface scratching during cold static initiation of the moving parts.

\section{Friction surface evaluation}

A series of tests were conducted on the Plint ring and liner simulator using production ring and cylinder liner segments at $100^{\circ} \mathrm{C}$ with step loading procedure. Figure 17 shows the results.

Figure 17 shows the effect of DLC on friction at $100^{\circ} \mathrm{C}$, the data are directly comparable to the data in Fig. 5. The bonded chemical film was able to lower the friction coefficient (COF) to below 0.002. If one assumes the COF of 0.06 as an indicator that the line is on the transition between EHL and hydrodynamic lubrication regime, DLC helps to bring about the early entry into hydrodynamic lubrication.

Friction reduction, theoretically, reduce the total energy

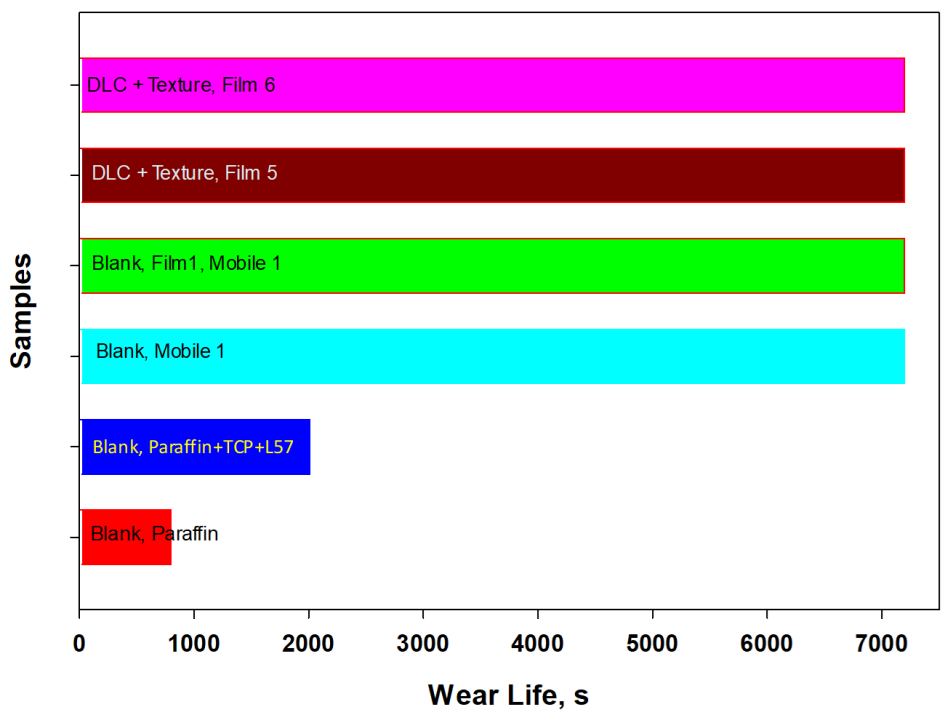

Fig. 15 Stage I test results for a specific combination of chemistry and textures, showing no failures for Mobil 1 lubricant and the 3 chemical films while the baseline blank in paraffin oil failed

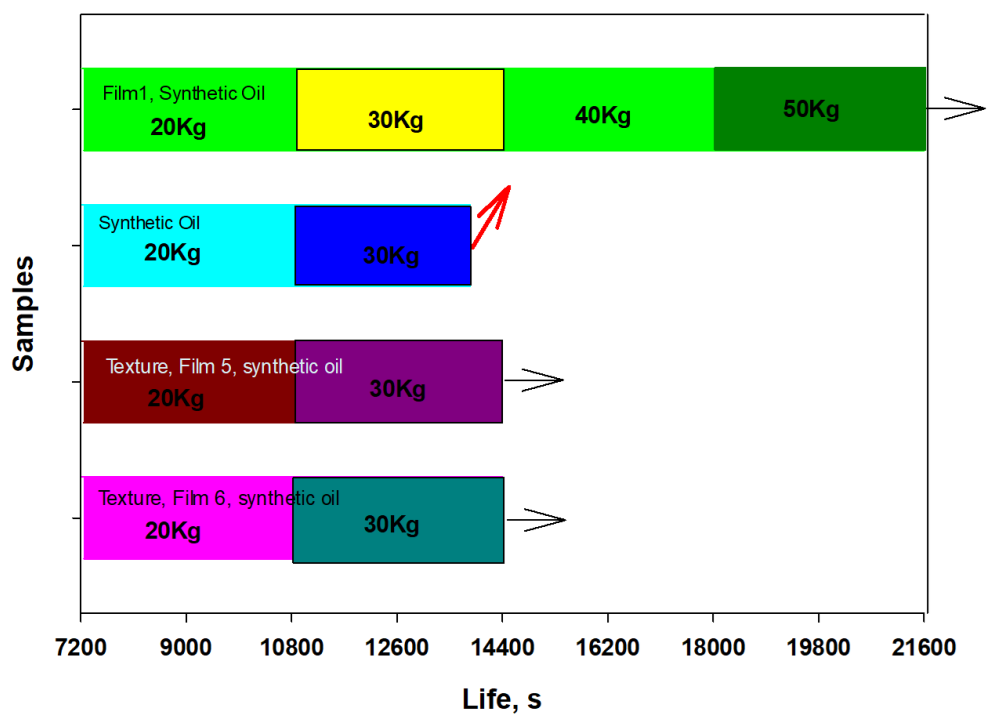

Fig. 16 Stage 2 durability test results showing times to failure data for the three bonded chemical films after they have gone through the previous 7000 seconds cycling testing 
consumption of the measurement system. Since the friction is measured using a ring and liner reciprocating test, one can use the difference in area under the curve to indicate the approximate effect of reduction in energy consumption, see Fig. 18.

In this example, we have demonstrated the basic design principle to systematically reduce friction of a complex system using various tools and techniques. There are many details in surface modification that will need elucidation in the future.

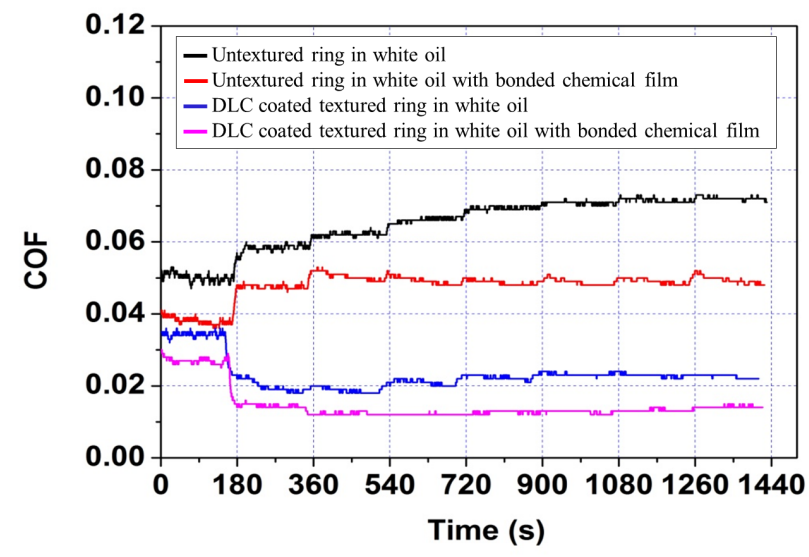

Fig. 17 Comparison of various treatments at $100^{\circ} \mathrm{C}$ using production ring and liner on the Plint tester
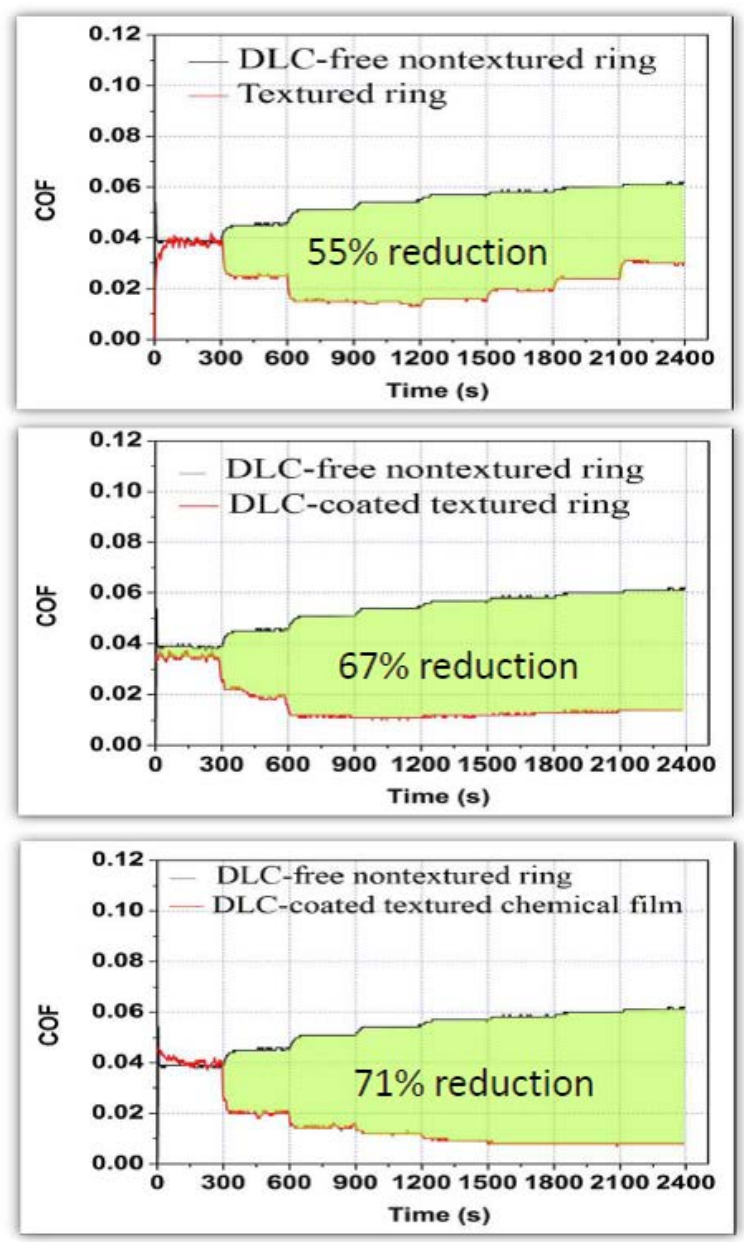

Fig. 18 Reduction of the area under the curve of friction as function of time, indicating reduction in energy

\section{Conclusions}

Surface and interface design is a new science that we begin to attempt, driven by our ability now to fabricate and control surface topography, directionality, and discrete surface textures. Coupled with buried sensors, actuators, and buried capsules, this area will grow rapidly to allow the design of self-adaptability, multifunctional attributes, and finally arrive at designing and fabricating smart surfaces, and intelligent systems.

\section{Acknowledgment}

The authors acknowledge the support from DOE Office of Vehicle Technology. The study is part of the IEA AMT TCP technical activity.

\section{References}

[1] Liu, G. Y. and Davis, U. C., Technical Report to NIST under a Cooperative Research Grant, 2000.

[2] Yang, S. H., Zhang, H. and Hsu, S. M., "Correction of Random Surface Roughness on Colloidal Probes in Measuring Adhesion," Langmuir 23, 3, 2007, 1195-1202.

[3] Bengisu, M. T. and Akay, A., "Relation of Dry-Friction to Surface Roughness," Transactions of ASME, Journal of Tribology, 119, 1, 1997, 18-25.

[4] Majumdar, B. C. and Hamrock, B. J., "Effect of Surface Roughness on Elastohydrodynamic Line Contact," Transactions of ASME, Journal of Lubrication Technology, 104, 3, 1982, 401-407.

[5] Jeng, Y. R., "Experimental Study of the Effects of Surface Roughness on Friction," Tribology Transactions, 33, 3, 1990, 402-410.

[6] Hsu, S. M., Ying, C. and Zhao, F., "The Nature of Friction: a Critical Assessment," Friction, 2, 1, 2014, 1-26.

[7] Fang, H. W., "Ultra-High Molecular Weight Polyethylene Wear Particle Effects on Bioactivity," Ph. D. Dissertation, University of Maryland, College Park, Maryland, 2004.

[8] Niederberger, S., Gracias, D. G., Komvopoulos, K. and Somorjai, G. A., "Transition from Nanoscale to Microscale Dynamic Friction Mechanisms on Polyethylene and Silicon Surfaces," Journal of Applied Physics, 87, 6, 2000, 3143-3150.

[9] Komvopoulos, K., Saka, N. and Suh, N. P., "The Mechanism of Friction in Boundary Lubrication," Transactions of ASME, Journal of Tribology, 107, 4, 1985, 452-462.

[10] Suh, M., Chae, Y., Kim, S., Hinoki, T. and Kohyama, A., "Effect of Geometrical Parameters in Micro-Grooved Crasshatch Pattern under Lubricated Sliding Friction," Tribology International, 43, 8, 2010, 1508-1517.

[11] Gropper, D., Wang, L. and Harvey, T. J., "Hydrodynamic Lubrication of Textured Surfaces: A Review of Modeling Techniques and Key Findings," Tribology International, 94, 2016, 509-529.

[12] Ma, C. and Zhu, H., "An Optimum Design Model for Textured Surface with Elliptical-Shape Dimples under Hydrodynamic Lubrication," Tribology International, 44, 9, 2011, 987-995.

[13] Al-Azizi, A. A., Eryilmaz, O., Erdemir, A. and Kim, S. H., “Effects of Nanoscale Surface Texture and Lubricant Molecular Structure on Boundary Lubrication in Liquid," Langmuir, 29, 44, 2013, 13419-13426.

[14] Hsu, S. M., Jing, Y. and Zhao, F., "Self-Adaptive Surface Texture Design for Friction Reduction Across the Lubrication Regimes," Surface Topography Metrology and Properties, 4, 1, 2015, 014004.

[15] Gachot, C., Rosenkranz, A., Hsu, S. M. and Costa, H. L., "A Critical Assessment of Surface Texturing for Friction and Wear Improvement," Wear, 372-373, 2017, 21-41. 